\title{
Outcomes of Descemet Membrane Endothelial Keratoplasty in Bangkok, Thailand
}

\author{
Kaevalin Lekhanont (iD) \\ Punyanuch Pisitpayat (iD) \\ Nontawat Cheewaruangroj' \\ Passara Jongkhajornpong (D)' \\ Manachai Nonpassopon (ID ${ }^{1}$ \\ Thunyarat Anothaisintawee ${ }^{2}$ \\ 'Department of Ophthalmology, Faculty \\ of Medicine Ramathibodi Hospital, \\ Mahidol University, Bangkok, Thailand; \\ ${ }^{2}$ Department of Family Medicine, Faculty \\ of Medicine Ramathibodi Hospital, \\ Mahidol University, Bangkok, Thailand
}

Correspondence: Kaevalin Lekhanont Department of Ophthalmology, Faculty of Medicine Ramathibodi Hospital, Mahidol University, Rama VI Road, Rajathevi, Bangkok, 10400, Thailand

Tel +662-20I- I560

Fax +662-20I-1516

Email lekhanont@yahoo.com
Purpose: To investigate the medium-term clinical outcomes and risk factors for primary graft failure after Descemet membrane endothelial keratoplasty (DMEK) in Thai patients.

Patients and Methods: This is a single-center retrospective cohort study. Sixty-two eyes of 62 patients who underwent DMEK at Ramathibodi Hospital, Bangkok, Thailand, with a minimum of 24-month follow-up were recruited. Preoperative donor and recipient characteristics, intraoperative data, and postoperative outcomes including best-corrected visual acuity (BCVA), graft clarity, endothelial cell density (ECD), central corneal thickness (CCT), and complications were evaluated at 1, 3, 6, 12, and 24 months after surgery.

Results: The mean age of the patients was $67.2 \pm 9.9$ years, and $52 \%$ were female. The mean follow-up time was $37.5 \pm 11.0$ months. The most common indications for DMEK were Fuchs' endothelial corneal dystrophy (FECD) (53.2\%) and pseudophakic bullous keratopathy (PBK) (17.7\%). Nearly half of patients had triple-DMEK. The median preoperative BCVA was 20/400. Postoperative BCVA of $\geq 20 / 40$ was reached in $37.1 \%$ and of $\geq$ $20 / 20$ in $6.5 \%$ after 1 month which increased to $54.8 \%$ and $17.7 \%$ after 3 months; and to $67.7 \%$ and $27.4 \%$ after 24 months. Endothelial cell loss (ECL) at 3, 6, 12, and 24 months was $30.5 \%, 33.8 \%, 44.4 \%$, and $45.9 \%$, respectively. Graft diameter was the single factor, showing a significant relationship with postoperative ECD. Most frequent postoperative complications included graft detachment (22.6\%), increased IOP/glaucoma (17.7\%), and primary graft failure (16.1\%). In univariate analysis, death-to-operation time and the diagnosis of PBK were significantly associated with the occurrence of primary graft failure.

Conclusion: DMEK is a safe, effective, and feasible treatment for endothelial failure in Asian eyes. Careful case selection, use of relatively fresh donor tissues, and appropriate surgical techniques can prevent primary graft failure and facilitate optimal outcomes following surgery.

Keywords: Descemet membrane endothelial keratoplasty, endothelial keratoplasty, Asian, outcomes, risk factors, complications

\section{Introduction}

Endothelial keratoplasty (EK) is the current standard of care for diseases of corneal endothelial dysfunction worldwide. Recent evidence supports that Descemet membrane endothelial keratoplasty (DMEK) is superior than Descemet stripping automated endothelial keratoplasty (DSAEK) in achieving a faster visual recovery, a better visual outcome, a lower immune rejection rate, and a less postoperative refractive error. ${ }^{1}$ Therefore, DMEK appears to be the surgical treatment of choice for EK in the United States and many countries in Europe, particularly for eyes with relatively normal anatomy. ${ }^{1,2}$ In Asia, although there has been a major shift from penetrating keratoplasty (PK) to 
EK for corneal endothelial failure in several countries, PK remained the preferred technique in some of them because of donor shortages, long waiting lists, and low patients' socioeconomic status. $^{3-9}$ By the time a patient is due for transplantation, EK might no longer be suitable because the corneal condition would often have deteriorated, resulting in significant central scarring. ${ }^{4}$ Additionally, DMEK is more technically challenging, requires dedicated training, and may involve a higher rebubbling rate than DSAEK during the early stages of the steep learning curve. ${ }^{1}$ Some surgeons tend to refuse to perform DMEK to avoid wasting valuable donor tissues. There are also some certain difficulties in performing DMEK on Asian eyes. ${ }^{10-12}$ Compared to Caucasians, Asians tends to have narrower palpebral fissures, smaller eyeballs with shallower anterior chambers, and darker irises. These unique characteristics could pose extra problems to surgeons regarding DMEK graft insertion, visualization, and orientation. ${ }^{10}$ Preexisting or newly created iris damage owing to DMEK also frequently occurred in Asian eyes with shorter axial length, potentially causing postoperative iris posterior synechiae and cystoid macular edema. ${ }^{11,12}$ Furthermore, a greater reduction of endothelial cells 6 months after surgery was observed in eyes with shorter axial length ($<24.0 \mathrm{~mm}$ ) compared to those with longer axial length $(>24.0 \mathrm{~mm}) .{ }^{13}$ As a result, DMEK has still not rapidly gained popularity in Asia, especially Southeast Asia. Previous studies investigating the safety and efficacy of DMEK were mainly from the United States and Europe. ${ }^{1}$ The outcomes of DMEK in Asia have been infrequently reported from India, Japan, Singapore, Turkey, and Nepal. ${ }^{14-23}$ In this study, we assessed the medium-term clinical results and risk factors for primary graft failure after DMEK in Thai patients with endothelial failure. To the best of our knowledge, there has been no study on risk factors for primary graft failure after DMEK.

\section{Materials and Methods Study Design}

A single-center retrospective cohort study was conducted at the Department of Ophthalmology, Ramathibodi Hospital, Bangkok, Thailand in accordance with the tenets of the Declaration of Helsinki. The study protocol was approved by the Institutional Review Board/Ethics Committee of Faculty of Medicine Ramathibodi Hospital,
Mahidol University. Written informed consent was obtained from all patients involved in the study.

\section{Study Population}

A retrospective chart review of 62 consecutive patients who underwent DMEK alone in pseudophakic eyes, as well as triple-DMEK (DMEK with simultaneous phacoemulsification cataract surgery) in phakic eyes with a minimum of 24-month follow-up were performed. Surgeries were done by 3 surgeons (K.L., P.J., M.N.) using the same technique. The very first 10 cases who had DMEK before 2015 were excluded from the study due to the initial learning-curve effects, use of different surgical technique, and insufficient data.

\section{Surgery}

A comprehensive history taking and an ophthalmological examination were performed to determine whether a patient was a good candidate for DMEK. Exclusion criteria included severe corneal edema with poor visibility of the anterior segment, significant corneal stromal opacities, concurrent keratoconus, extensive peripheral anterior synechiae, uncontrolled glaucoma, presence of anterior chamber intraocular lens (IOL), vitreous, or glaucoma drainage device in the anterior chamber, large pupils, obvious iris abnormalities or aniridia, aphakia, preexisting vision-limiting posterior segment comorbidities, and amblyopia.

\section{DMEK Graft Preparation}

The donor corneoscleral button, stored in Optisol-GS (Bausch \& Lomb, Rochester, NY) at $4{ }^{\circ} \mathrm{C}$ were provided by either the Thai Red Cross Eye Bank (19 tissues) or Eye Bank Association of America (EBAA)-accredited facilities in the United States (43 tissues). ${ }^{24}$ The donor age was between 50 and 75 years with an endothelial cell density (ECD) of at least 2700 cells $/ \mathrm{mm}^{2}$ DMEK donor graft was prepared by the operating surgeon in the operation room directly prior to surgery. Pre-stripped and pre-stamped or pre-loaded tissue were unavailable in Thailand. Briefly, the corneoscleral button was first placed endothelial side up on a Teflon cutting block. Descemet membrane (DM) was scored at the periphery just inside the trabecular meshwork using a blunt-tip Sinskey hook. After scoring was completed, the scored edge was stained with $0.05 \%$ trypan blue solution (1:3 dilution of $0.15 \%$ MembraneBlue ${ }^{\circledR}$, DORC International, Zuidland, The Netherlands) for 10 seconds to aid visualization. The scored edge was lifted 
$360^{\circ}$ using a blunt-tip Sinskey hook under balanced salt solution (BSS). The lifted edge was re-stained with $0.05 \%$ trypan blue solution for 10 seconds and rinsed with BSS. The irregular DM tags along the scored edge were removed. Partial peeling of the DM by quadrants was performed $360^{\circ}$ using McPherson forceps under BSS. The DM graft was kept immersed in BSS ready for the trephination and final peel.

\section{Recipient Eye Preparation}

All surgeries were performed with a temporal approach under general anesthesia. Acetazolamide was given to all patients shortly before surgery to lower the positive vitreous pressure in order to obtain an appropriately soft eye. A urethral catheter was inserted. In pseudophakic eyes, the pupil was constricted using $2 \%$ pilocarpine eye drops. In phakic eyes, the pupil was dilated using $2.5 \%$ phenylephrine for triple-DMEK. In the case of triple-DMEK, a phacoemulsification with implantation of an acrylic IOL into the capsular bag was performed prior to DMEK. The refractive target of -0.50 diopters (D) and $-1.00 \mathrm{D}$ were chosen for DMEK alone and triple-DMEK respectively, based on previously published data. ${ }^{25-28}$

\section{Surgical Technique}

In brief, depending on the corneal diameter and type of pathology, a 7.0 to $8.5-\mathrm{mm}$, interrupted dot, circular mark was made on the corneal epithelial surface, using a fine-tip mark pen, to guide the DM stripping of the recipient cornea. Two paracenteses, which were 4 clock hours away from each other, and 3.0-mm main incision (clear corneal tunnel incision) in the center of them, were created temporally. These incisions must have not been too centered to avoid an overlap between the internal openings of wounds and the DM graft. Cohesive viscoelastic agent (sodium hyaluronate) was injected into the anterior chamber. Descemetorhexis was performed using a reverse Sinskey's hook, with a diameter approximately $0.5 \mathrm{~mm}$ larger than the planned diameter of the DM graft to prevent an overlap between the donor and recipient DMs. Loose DM tags were removed using Micro-Surgical Technology (MST) forceps. The viscoelastic agent was washed out and the anterior chamber was refilled with BSS. Intracameral carbachol $\left(\right.$ Miostat $^{\mathbb{B}}$, Alcon Laboratories Inc., USA) injection was done to constrict the pupil in triple-DMEK cases. A small but patent inferior surgical peripheral iridectomy was performed at 6 o'clock. No laser peripheral iridotomy was made preoperatively in our series. The anterior chamber was irrigated until it was clear, followed by temporary air tamponade to stop oozing of blood from peripheral iridectomy if any and minimize further corneal edema after descemetorhexis while loading the DM graft.

A 7.0 to $8.5-\mathrm{mm}$ partial trephination of the cornea donor was made. The DM graft was completely peeled off the posterior stroma and stained with $0.1 \%$ trypan blue solution ( $2: 3$ dilution of $0.15 \%$ MembraneBlue $^{\circledR}$ ) for 3 minutes. Because a dark iris in an Asian eye could make intraoperative graft visualization difficult, the concentration of trypan blue used for the final stain in this study was $0.1 \%$ rather than $0.05 \%$ or typical concentration of $0.06 \%$ to improve the visibility of the DM graft during surgery. Staining of DM graft with $0.1 \%$ trypan blue up to 4 minutes theoretically allowed more intense stain without additional adverse effects as the use of the higher concentration of trypan blue $\left(0.15 \%\right.$ MembraneBlue $\left.{ }^{\circledR}\right)$ was proven to be effective and safe for staining DMEK donor tissue for 3-4 minutes. ${ }^{29,30}$ Stained DM roll was aspirated into the curved glass injector system (DMEK surgical disposable set, DORC International ${ }^{\circledR}$ Zuidland, The Netherlands) ready for injection into the recipient eye.

The air in the anterior chamber was replaced with BSS. The donor DM roll was injected via the main incision to the anterior chamber. The main wound was closed immediately with a 10-0 nylon suture after the injector came out. The DM roll was manipulated meticulously and gently using no-touch tapping and fluid wave techniques until it was opened in the correct orientation. ${ }^{31,32}$ A handheld slit beam and endoilluminator were used intraoperatively to determine the graft orientation. ${ }^{33}$ The DM graft was then centered and fully unscrolled. Air was injected underneath the center of the DM graft using a 30gauge blunt cannula to attach the graft to the recipient stroma bed. The anterior chamber was completely filled with $20 \%$ SF6 gas. All wounds were checked to ensure watertight closure with appropriate ocular tension. The patient was instructed to strictly lie flat on their head and back in the supine position for at least 2 hour.

\section{Postoperative Care}

Two hour after surgery, the patient was examined under slit-lamp to assess the intraocular pressure (IOP), the size of the air bubble, the coverage of the peripheral iridectomy by the bubble in the upright position, and the plane of the iris and IOL. A postoperative regimen included topical $1 \%$ prednisolone acetate every 2 hours, $0.5 \%$ levofloxacin 4 
times daily and lubricants 4 times daily for 4 weeks, followed by tapering doses of topical \% prednisolone acetate to once daily by 6 months, and thereafter continued indefinitely. Steroid responders received anti-glaucoma medications and $1 \%$ prednisolone acetate was changed to fluorometholone as needed.

All patients were hospitalized and asked to keep a strict supine position postoperatively for at least 3 days. They were examined daily until the residual air bubble did not cover the pupil when they sat up and there was no corneal epithelial defect, and then discharged from the hospital. Follow-up examinations were performed at 1, 3, 6, 9, and 12 months after surgery and then every 6 months thereafter.

\section{Outcome Measures}

The main outcome measures were postoperative bestcorrected visual acuity (BCVA), graft clarity, ECD, central corneal thickness (CCT), and complications. The secondary outcomes were the relationships between the occurrence of primary graft failure as well as postoperative ECD and various donor and recipient characteristics including donor age, donor ECD, death-to-preservation time, death-to-operation time, recipient age, sex, preoperative diagnoses, preoperative BCVA, axial length, type of surgery, graft diameter, intraoperative complications. Management of postoperative complications were recorded in all patients.

Preoperative donor ECD was evaluated by provider eye banks in Thailand and the United States using specular microscopy. Postoperative ECD was determined by noncontact specular microscopy (EM-3000; Tomey, Nagoya, Japan) in our clinic at 3, 6, 12 and 24 months after surgery. The central corneal endothelium was photographed, at least 50-100 cells were counted for each image, and the postoperative ECD was calculated. ${ }^{22}$ CCT values was measured by high-resolution rotating Scheimpflug imaging (Pentacam HR; Oculus, Wetzlar, Germany). Anterior segment optical coherence tomography (AS-OCT) (Visante OCT; Carl Zeiss Meditec, Dublin CA, USA) was performed postoperatively in eyes with suspected DM detachment.

\section{Statistical Analysis}

Statistical analyses were performed with the statistical software package STATA version 15.0 (Stata Corp, College Station, Texas, USA). Continuous data were expressed as either mean \pm standard deviation (SD) or median and range, depending on the normality of distribution. These were compared using independent $t$ test or Mann-Whitney $U$-test. Categorical data described as frequency and percentage, were analyzed with Chi-square or Fisher's exact tests. Changes in BCVA and ECD over time from preoperative to 24-month follow-up were assessed using a multilevel linear mixed regression model. The time variable was considered as fixed effect model, while the subject variable was considered as random effect model. Associations between primary graft failure and various donor or recipient characteristics were evaluated using logistic regression analysis. $P$-values less than 0.05 were considered to be statistically significant.

\section{Results}

\section{Patient Demographics}

A total 62 eyes of 62 Thai patients were included in this study. All of them were of Asian ethnicity. The mean age of the patients was $67.2 \pm 9.9$ years (range, 48-86 years) and $52 \%$ were female. The mean follow-up time was 37.5 \pm 11.0 months (range, 48-59 months). The most common indication for DMEK in this series was Fuchs' endothelial corneal dystrophy (FECD) (53.2\%), followed by pseudophakic bullous keratopathy (PBK) (17.7\%) and corneal decompensation caused by viral endotheliitis (9.7\%) (Table 1). Thirty-three patients (53.2\%) underwent DMEK alone and 29 patients (46.8\%) had triple-DMEK. The median preoperative BCVA was 20/400 (range, 20/40counting fingers). The average axial length was $23.30 \pm$ $1.0 \mathrm{~mm}$, ranging from 20.82 to $25.54 \mathrm{~mm}$. Details of patient demographics are shown in Table 1.

\section{Donor Characteristics}

The mean donor age was $63.0 \pm 6.5$ years (range, 50-75 years). A total of $56.5 \%$ of donor tissues were from male donors, $38.7 \%$ were from female, and the donor sex of the remaining $4.8 \%$ was not reported (imported corneas). No donor tissues had a history of diabetes mellitus. All grafts were from phakic donors. The mean enucleation-topreservation time was $8.9 \pm 5.9$ hours (range, 1.2-23 hours) and the mean death-to-surgery time was $7.6 \pm 2.5$ days (range, 2-12 days). According to the eye banks, the mean postharvesting ECD was $2931 \pm 178$ cells $/ \mathrm{mm}^{2}$ (range, 2703-3333 cells $/ \mathrm{mm}^{2}$ ). The median DMEK graft diameter used was $8.0 \mathrm{~mm}$ (range, $7.0-8.5 \mathrm{~mm}$ ). Table 2 summarizes the donor information. 
Table I Recipient Demographics (62 Patients)

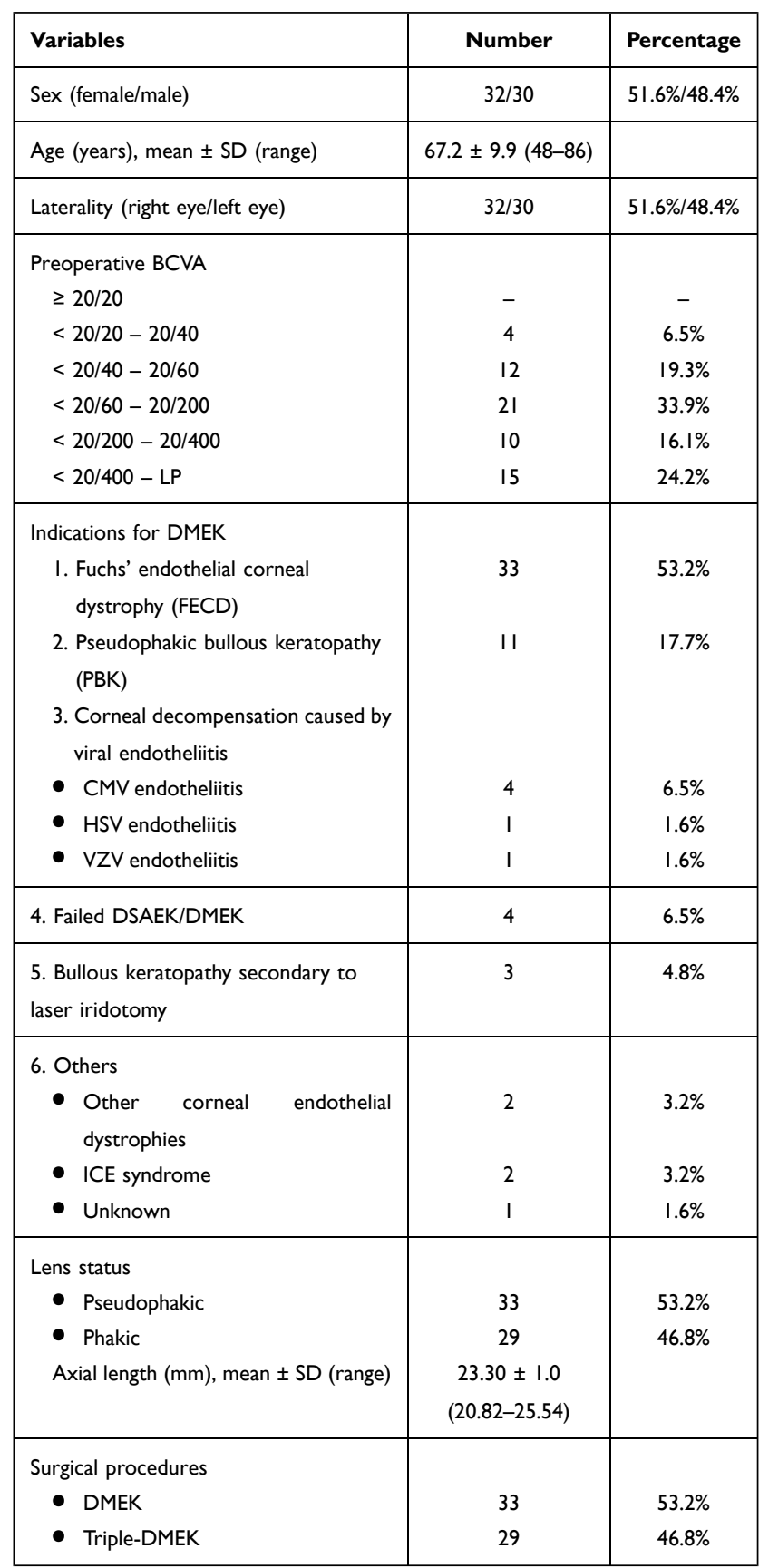

Abbreviations: BCVA, best-corrected visual acuity; CMV, cytomegalovirus; DMEK, Descemet membrane endothelial keratoplasty; DSAEK, Descemet stripping automated endothelial keratoplasty; HSV, herpes simplex virus; ICE, iridocorneal endothelial; LP, light perception; SD, standard deviation; VZV, varicella zoster virus.

\section{Intraoperative Complications}

Intraoperative complications were encountered in 3 cases (4.8\%). Two of them had graft ejection from the eye through the main wound during graft insertion because the anterior chamber was over-pressurized. Fortunately, the same graft was retrieved, reloaded, and reinjected into the anterior chamber successfully. The surgery was continued uneventfully.
Table 2 Donor Characteristics (62 Donor Tissues)

\begin{tabular}{|l|c|c|}
\hline Variables & Number & Percentage \\
\hline Sex (female/male) & $\begin{array}{c}24 / 35 \text { (No data of } \\
\text { the rest 3 donors) }\end{array}$ & $38.7 / 56.5 \%$ \\
\hline Age (years), mean \pm SD (range) & $63.0 \pm 6.5(50-75)$ & \\
\hline Mean enucleation-to- & $8.9 \pm 5.9(1.2-23)$ & \\
preservation time (hours), & & \\
mean \pm SD (range) & & \\
\hline Mean death-to-operation time & $7.6 \pm 2.5(2-12)$ & \\
(days), mean \pm SD (range) & & \\
\hline Mean post-harvesting ECD & $2931 \pm 178$ & \\
(cells/mm ${ }^{2}$ ) mean \pm SD (range) & $(2703-3333)$ & \\
\hline Graft diameter (mm) & & $3.2 \%$ \\
-7.00 & 2 & $12.9 \%$ \\
-7.50 & 8 & $67.7 \%$ \\
-8.00 & 42 & $12.9 \%$ \\
-8.25 & 2 & \\
-8.50 & 8 & \\
\hline
\end{tabular}

Abbreviation: SD, standard deviation.

Excessive graft manipulation was done in another patient who had pre-existing corectopia and scattered peripheral anterior synechiae, making graft unfolding and positioning difficult. No donor tissue was lost during DMEK preparation.

\section{Visual Outcomes}

Preoperative mean BCVA of $1.03 \pm 0.53$ (range, 0.30-1.90) $\log$ MAR improved to $0.42 \pm 0.58$ (range, $0.00-2.20$ ) $\log$ MAR at 24 months after DMEK $(P<0.001)$. A BCVA of $\geq 20 / 40$ was reached in $37.1 \%$ of eyes $(23 / 62)$ and of $\geq$ $20 / 20$ in $6.5 \%$ of eyes $(4 / 62)$ after 1 month which increased to $54.8 \%(34 / 62)$ and $17.7 \%(11 / 62)$ after 3 months; and to $67.7 \%(42 / 62)$ and $27.4 \%(17 / 62)$ after 12 months. Improvement in BCVA remained stable up to 24 months after surgery.

For further analysis, 11 eyes with primary or secondary graft failure were excluded. From a total of 51 eyes with a functional graft and normal visual potential, 78.4\% (40/ 51) achieved a BCVA of $\geq 20 / 40$ and 33.3\% (17/51) achieved $\geq 20 / 20$ at 24 months after surgery (Table 3 ).

\section{Endothelial Cell Density}

The average preoperative ECD was $2931 \pm 178 \mathrm{cell} / \mathrm{mm}^{2}$ (range, 2703-3333 cell $/ \mathrm{mm}^{2}$ ). In 51 eyes with a clear graft, ECD decreased to $2038 \pm 468 \mathrm{cell} / \mathrm{mm}^{2}$ at 3 months, $1941 \pm 425 \mathrm{cell} / \mathrm{mm}^{2}$ at 6 months, $1629 \pm 408 \mathrm{cell} / \mathrm{mm}^{2}$ at 
Table 3 Best-Corrected Visual Acuity, Endothelial Cell Density, and Central Corneal Thickness at 3, 6, I2, and 24 Months After DMEK

\begin{tabular}{|c|c|c|c|c|c|c|}
\hline Clinical Outcomes & Preoperative & I Month & 3 Months & 6 Months & 12 Months & 24 Months \\
\hline \multicolumn{7}{|l|}{ BCVA including all eyes (62 eyes) } \\
\hline$\geq 20 / 20$ & - & $6.5 \%$ & $17.7 \%$ & $27.4 \%$ & $27.4 \%$ & $27.4 \%$ \\
\hline$<20 / 20-20 / 25$ & - & $6.5 \%$ & $14.5 \%$ & $19.4 \%$ & $22.6 \%$ & $22.6 \%$ \\
\hline$<20 / 25-20 / 40$ & $6.5 \%$ & $24.2 \%$ & $22.6 \%$ & $21.0 \%$ & $17.7 \%$ & $17.7 \%$ \\
\hline$<20 / 40-20 / 60$ & $19.3 \%$ & $27.4 \%$ & $16.1 \%$ & $6.5 \%$ & $6.5 \%$ & $6.5 \%$ \\
\hline$<20 / 60-20 / 200$ & $33.9 \%$ & $24.2 \%$ & $16.1 \%$ & $12.9 \%$ & $12.9 \%$ & $12.9 \%$ \\
\hline$<20 / 200-20 / 400$ & $16.1 \%$ & $6.5 \%$ & $6.5 \%$ & $4.8 \%$ & $4.8 \%$ & $4.8 \%$ \\
\hline$<20 / 400-\mathrm{LP}$ & $24.2 \%$ & $4.8 \%$ & $6.5 \%$ & $8.1 \%$ & $8.1 \%$ & $8.1 \%$ \\
\hline \multicolumn{7}{|l|}{ BCVA excluding eyes with graft failure (5I eyes) } \\
\hline$\geq 20 / 20$ & - & $7.8 \%$ & $21.6 \%$ & $33.3 \%$ & $33.3 \%$ & $33.3 \%$ \\
\hline$<20 / 20-20 / 25$ & - & $7.8 \%$ & $17.6 \%$ & $23.5 \%$ & $25.5 \%$ & $25.5 \%$ \\
\hline$<20 / 20-20 / 40$ & $5.9 \%$ & $29.4 \%$ & $27.5 \%$ & $21.6 \%$ & $19.6 \%$ & $19.6 \%$ \\
\hline$<20 / 40-20 / 60$ & $23.5 \%$ & $31.6 \%$ & $15.7 \%$ & $7.8 \%$ & $7.8 \%$ & $7.8 \%$ \\
\hline$<20 / 60-20 / 200$ & $37.3 \%$ & $23.5 \%$ & $17.6 \%$ & $13.7 \%$ & $13.7 \%$ & $13.7 \%$ \\
\hline$<20 / 200-20 / 400$ & $11.8 \%$ & - & - & - & - & - \\
\hline$<20 / 400-\mathrm{LP}$ & $21.6 \%$ & - & - & - & - & - \\
\hline $\mathrm{ECD}$, mean $\pm \mathrm{SD}\left(\right.$ cell $\left./ \mathrm{mm}^{2}\right)(5 \mathrm{l}$ eyes $)$ & $2931 \pm 178$ & & $2038 \pm 468$ & $|94| \pm 425$ & $1629 \pm 408$ & $|586 \pm 35|$ \\
\hline Mean ECL & & & $30.5 \%$ & $33.8 \%$ & $44.4 \%$ & $45.9 \%$ \\
\hline $\mathrm{CCT}$, mean $\pm \mathrm{SD}(\mu \mathrm{m})(5 \mathrm{I}$ eyes $)$ & $721 \pm 91$ & & $507 \pm 73$ & $496 \pm 67$ & $481 \pm 44$ & $484 \pm 45$ \\
\hline Mean CCT decrease & & & $29.7 \%$ & $31.2 \%$ & $33.3 \%$ & $32.7 \%$ \\
\hline
\end{tabular}

Abbreviations: BCVA, best-corrected visual acuity; CCT, central corneal thickness; DMEK, Descemet membrane endothelial keratoplasty; ECD, endothelial cell density; $E C L$, endothelial cell loss; SD, standard deviation.

12 months, and $1586 \pm 351 \mathrm{cell} / \mathrm{mm}^{2}$ at 24 months postoperatively, corresponding to an endothelial cell loss (ECL) of $30.5 \%, 33.8 \%, 44.4 \%$, and $45.9 \%$, respectively. The decrease of ECD when compared to preoperative values and between each time point was statistically significant $(P<0.005$ for all time points). Donor age, donor $\mathrm{ECD}$, donor storage time, patient age, patient sex, preoperative diagnosis, preoperative BCVA, axial length, type of surgery, and postoperative graft detachment were not significantly related to postoperative ECD, whereas graft diameter showed a significant association (Table 4).

\section{Central Corneal Thickness}

The mean preoperative CCT was $721 \pm 91 \mu \mathrm{m}$ (range, 616-1007 $\mu \mathrm{m})$. In 51 eyes with a clear graft, CCT decreased to $507 \pm 73 \mu \mathrm{m}, 496 \pm 67 \mu \mathrm{m}, 481 \pm 44 \mu \mathrm{m}$, and $485 \pm 45 \mu \mathrm{m}$ at 3, 6, 12, 24 months respectively, corresponding to an overall decrease of $29.7 \%, 31.2 \%$, $33.3 \%$, and $32.7 \%$ respectively. The decrease of CCT within the first 3 months was statistically significant $(P<$
0.005). In the follow-up until 24 months, no further statistically significant change was observed.

\section{Postoperative Complications}

Postoperative complications included graft detachment, primary graft failure, increased IOP/glaucoma, cystoid macular edema, endothelial graft rejection, epiretinal membrane, viral endotheliitis, and filamentary keratitis (Table 5). Graft detachment (14 eyes; 22.6\%) was the most common early postoperative complication in this series. All graft detachments appeared within 10 days after surgery. Approximately $60 \%$ of these cases $(8 / 14$ eyes) showed a partial graft detachment in $<1 / 3$ of graft surface area and only 3 of 8 cases needed re-bubbling with air at the slit-lamp. Spontaneous resolution with time was noted in the remaining patients with peripheral graft detachment. Detachments involving over $1 / 3$ of graft surface area occurred in 6 cases and 5 cases were treated with re-bubbling. One case failed repeat re-bubbling and developed total graft detachment, requiring re-DMEK 2 months 
Table 4 Effects of the Donor and Recipient Variables from the Multilevel Linear Mixed Regression Models for Postoperative Endothelial Cell Density (ECD)

\begin{tabular}{|c|c|c|c|}
\hline \multirow[t]{2}{*}{ Variables } & \multicolumn{3}{|c|}{ ECD } \\
\hline & Coef. & $95 \% \mathrm{Cl}$ & $P$ \\
\hline \multicolumn{4}{|l|}{ Univariate analysis } \\
\hline Donor age & -0.30 & $-10.13-9.53$ & 0.952 \\
\hline Donor ECD & 0.10 & $-0.17-0.37$ & 0.467 \\
\hline Death-to-operation time & 22.87 & $-1.95-47.72$ & 0.071 \\
\hline Patient age & -4.29 & $-10.40-1.82$ & 0.169 \\
\hline Patient sex & -44.08 & $-167.65-79.50$ & 0.484 \\
\hline \multicolumn{4}{|l|}{ Original diagnosis } \\
\hline - FECD vs PBK & 53.49 & $-127.80-234.78$ & 0.563 \\
\hline $\begin{array}{l}\text { - FECD vs corneal } \\
\text { decompensation caused } \\
\text { by viral endotheliitis }\end{array}$ & -134.74 & $-357.16-87.69$ & 0.235 \\
\hline - FECD vs failed EK & 170.76 & $-92.43-433.96$ & 0.204 \\
\hline - FECD vs BK due to L-PI & -224.20 & $-535.87-87.46$ & 0.159 \\
\hline - FECD vs others & 36.59 & $-180.96-254.13$ & 0.742 \\
\hline Preoperative BCVA & -2.73 & $-148.86-143.39$ & 0.971 \\
\hline Axial length & 43.27 & $-21.29-107.84$ & 0.189 \\
\hline Type of surgery & -36.54 & $-159.95-86.87$ & 0.562 \\
\hline Graft diameter & 267.47 & $65.83-469.12$ & 0.009 \\
\hline $\begin{array}{l}\text { Postoperative graft } \\
\text { detachment }\end{array}$ & -91.99 & $-253.64-69.66$ & 0.265 \\
\hline Mutivariate analysis* & & & \\
\hline Death-to-operation time & 19.62 & $-5.07-44.31$ & 0.119 \\
\hline Graft diameter & 250.32 & $48.19-452.45$ & 0.015 \\
\hline
\end{tabular}

Notes: *Variables with $\mathrm{p}$-value $<0.1$ in the univariate analysis were included in the multivariate model. Bold values indicate statistical significance with a $p$-value less than 0.05 .

Abbreviations: BCVA, best-corrected visual acuity; $\mathrm{BK}$, bullous keratopathy; $\mathrm{Cl}$, confidence interval; Coef., regression coefficients mixed effects; $E C D$, endothelial cell density; EK, endothelial keratoplasty; FECD, Fuchs' endothelial corneal dystrophy; PBK, pseudophakic bullous keratopathy; Pl, peripheral iridotomy.

later. Another case had an upside-down graft, requiring secondary graft reposition.

The second most common early postoperative complication was primary graft failure (10 eyes; 16.1\%). A second keratoplasty was performed in 5 cases (DSAEK 2 cases, DMEK 2 case, and PK 1 case). The other patients were still on the waiting list for regrafting due to the inadequate supply of donor corneas. Univariate analysis revealed that death-to-operation time and the diagnosis of PBK were significantly associated with the occurrence of primary graft failure (Table 6). However, the multivariate analysis to adjust for many
Table 5 Postoperative Complications and Additional Surgical Interventions After DMEK

\begin{tabular}{|c|c|c|}
\hline Complications & Number & Percentage \\
\hline Graft detachment & 14 & $22.6 \%$ \\
\hline - Minor (< I/3 of graft surface area) & 8 & $12.9 \%$ \\
\hline - Major (> I/3 of graft surface area) & 6 & $9.7 \%$ \\
\hline Graft failure & 11 & $17.7 \%$ \\
\hline - Primary & 10 & $16.1 \%$ \\
\hline - Secondary & 1 & $1.6 \%$ \\
\hline Increased IOP/glaucoma & II & $17.7 \%$ \\
\hline - Steroid-related ocular hypertension & 8 & $12.9 \%$ \\
\hline Cystoid macular edema & 3 & $4.8 \%$ \\
\hline Allograft rejection & 3 & $4.8 \%$ \\
\hline Epiretinal membrane & 2 & $3.2 \%$ \\
\hline Pupillary block & 1 & $1.6 \%$ \\
\hline CMV endotheliitis & 1 & $1.6 \%$ \\
\hline Filamentary keratitis & 1 & $1.6 \%$ \\
\hline Additional surgical interventions & Number & Percentage \\
\hline Re-bubbling & 8 & $12.9 \%$ \\
\hline Re-transplantation & 3 & $4.8 \%$ \\
\hline • DSAEK & 2 & $3.2 \%$ \\
\hline - DMEK & 1 & $1.6 \%$ \\
\hline Graft reposition & 1 & $1.6 \%$ \\
\hline Pars plana vitrectomy & 1 & $1.6 \%$ \\
\hline
\end{tabular}

Abbreviations: CMV, cytomegalovirus; DMEK, Descemet membrane endothelial keratoplasty; DSAEK, Descemet stripping automated endothelial keratoplasty; IOP, intraocular pressure.

confounding variables could not be applied due to the small number of cases.

The most frequent late postoperative complication was IOP elevation (11 eyes; 17.7\%). Three eyes had preexisting primary angle closure glaucoma and one of them had prior trabeculectomy. Eight patients developed steroidrelated ocular hypertension. All of them were successfully medically managed by initiating or increasing topical antiglaucoma medications, or reducing the steroid strength or dosing frequency.

Cystoid macular edema was diagnosed in 3 cases using spectral-domain optical coherence tomography (SD-OCT) (Spectralis OCT; Heidelberg Engineering, Heidelberg, Germany). One of them had diabetes mellitus. The diabetic patient developed cystoid macular edema secondary 
Table 6 Factors Associated with Primary Graft Failure After DMEK by Univariate Analysis

\begin{tabular}{|c|c|c|c|}
\hline \multirow[t]{2}{*}{ Variables } & \multicolumn{3}{|c|}{ Primary Graft Failure } \\
\hline & OR & $95 \% \mathrm{Cl}$ & $P$ \\
\hline \multicolumn{4}{|l|}{ Univariate analysis } \\
\hline Donor age & 0.97 & $0.85-1.11$ & 0.653 \\
\hline Donor ECD & 0.999 & $0.995-1.002$ & 0.533 \\
\hline Death-to-operation time & 2.05 & $1.06-3.96$ & 0.033 \\
\hline Patient age & 1.00 & $0.94-1.07$ & 0.906 \\
\hline Patient sex & 0.47 & $0.12-1.80$ & 0.271 \\
\hline \multicolumn{4}{|l|}{ Original diagnosis } \\
\hline - FECD vs PBK & 12.92 & $2.01-82.83$ & 0.007 \\
\hline $\begin{array}{l}\text { - FECD vs corneal decompensa- } \\
\text { tion caused by viral } \\
\text { endotheliitis }\end{array}$ & 7.75 & $0.84-71.31$ & 0.071 \\
\hline - FECD vs failed EK & 5.17 & $0.36-75.13$ & 0.229 \\
\hline - FECD vs BK due to L-PI & 7.75 & $0.47-126.69$ & 0.151 \\
\hline - FECD vs others & 1 & - & - \\
\hline Preoperative BCVA & 4.81 & $0.86-27.04$ & 0.074 \\
\hline Axial length & 0.84 & $0.43-1.64$ & 0.616 \\
\hline Type of surgery & 0.36 & $0.09-1.52$ & 0.164 \\
\hline Graft diameter & 0.59 & $0.08-4.41$ & 0.609 \\
\hline $\begin{array}{l}\text { Presence of intraoperative } \\
\text { complications }\end{array}$ & 1.00 & - & - \\
\hline
\end{tabular}

Note: Bold values indicate statistical significance with a $p$-value less than 0.05 . Abbreviations: BCVA, best-corrected visual acuity; $\mathrm{BK}$, bullous keratopathy; $\mathrm{Cl}$, confidence interval; DMEK, Descemet membrane endothelial keratoplasty; ECD, endothelial cell density; EK, endothelial keratoplasty; FECD, Fuchs' endothelial corneal dystrophy; OR, odd ratio; PBK, pseudophakic bullous keratopathy; PI, peripheral iridotomy.

to hemi-retinal vein occlusion 7 months following triple DMEK. It responded well to intravitreal anti- vascular endothelial growth factor (anti-VEGF) therapy. The other 2 cases had cystoid macular edema detected at 1 month after DMEK alone. It was hard to determine whether its onset was before or after surgery because preoperative macular OCT-imaging was not routinely performed. One patient had epiretinal membrane along with cystoid macular edema and noted a gradual gain in BCVA after pars plana vitrectomy with membrane peel. Another cystoid macular edema disappeared with topical steroids and nonsteroidal anti-inflammatory agents, followed by excellent visual recovery.

Endothelial allograft rejection was found in 3 patients. All of them developed the rejection episode within the first 9 months and was successfully treated with topical and oral steroids. Secondary graft failure occurred in 1 eye because of recurrent $\mathrm{CMV}$ endotheliitis 3 months after surgery.

\section{Discussion}

Today, there is substantial evidence that DMEK is a safe and effective treatment for corneal endothelial failure. However, the conclusion was drawn majorly from the studies in the US and European countries. ${ }^{1}$ Although there have recently been more reports on case series after DMEK in Asian countries, most of them addressed the issues of surgical techniques and complications that should be specifically concerned with when performing DMEK in Asian eyes. ${ }^{10-12-15-18-19}$ Only few reports analyzed the overall clinical outcomes of DMEK in a large number of patients (more than 50 cases) with varying follow-up time. ${ }^{14,16,19-21}$ Table 7 demonstrates the summary of large studies (4 retrospective and 1 prospective) evaluating the outcomes of DMEK in Asia, including this study.

In our retrospective cohort study, we evaluated the medium-term clinical outcomes of 62 Thai patients who underwent DMEK with a follow-up of 2 years and identified which parameters may affect the surgical outcomes. This study also highlighted the results of DMEK using imported donor corneas. Our data confirmed that visual acuity improved dramatically within a few months, became stable by month 6 , and was maintained up to 24 months after surgery in uncomplicated cases. Approximately $18 \%$ and $27 \%$ of eyes achieved a postoperative BCVA of $\geq 20 / 20$ at 3 and 6 months respectively. If the eyes with primary graft failure or progressive graft detachment requiring regrafts were excluded, these figures would have increased to $22 \%$ and $33 \%$. The visual outcomes in Thai patients were similar to previously published data which revealed a BCVA of $\geq 20$ / 20 in $29-32 \%$ and $17-67 \%$ of patients at 3 and 6 months postoperatively, respectively. ${ }^{1}$ Nonetheless, several western studies showed better results, indicating that $41-67 \%$ of DMEK eyes achieved BCVA of $\geq 20 / 20$ at 6 months after surgery. ${ }^{1,34}$ This could be explained by a different patient population in our series with most cases presenting with advanced FECD and PBK; and 40.3\% having preoperative BCVA of $<20 / 200-$ LP. Long-standing corneal edema leading to some degree of subepithelial and stromal scarring and vascularization can result in suboptimal postoperative BCVA despite good graft adhesion, centration, and function.

The 2-year ECL in our series was $46 \%$ with a more rapid decline occurring in the first few months after 


\begin{tabular}{|c|c|c|c|c|c|}
\hline 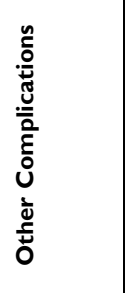 & 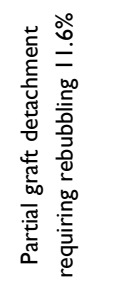 & 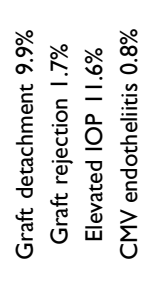 & 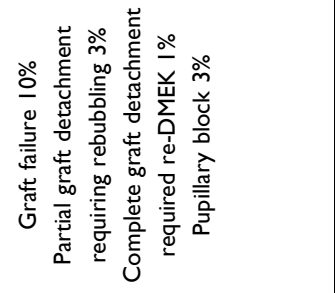 & 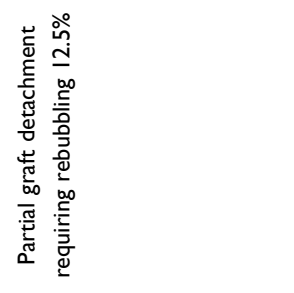 & 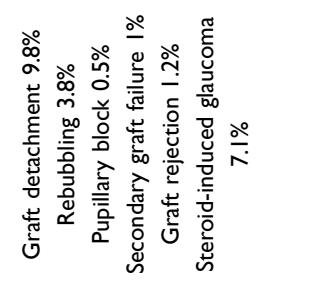 \\
\hline 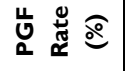 & $\stackrel{n}{\underline{n}}$ & $\overleftarrow{\Sigma}$ & $\overleftarrow{\Sigma}$ & 0 & นุ \\
\hline ư & 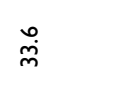 & $\overleftarrow{z}$ & 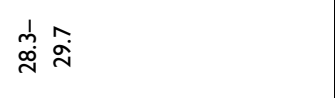 & 芦 & 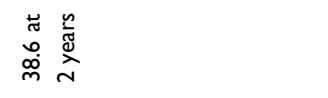 \\
\hline 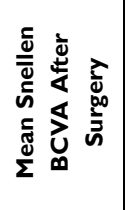 & 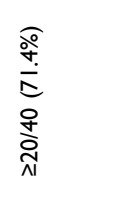 & $\overleftarrow{z}$ & $\stackrel{\widetilde{N}}{\stackrel{\sim}{\sim}}$ & ָั & 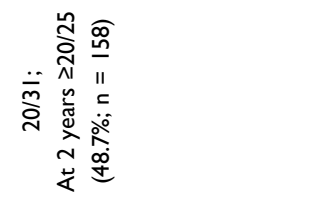 \\
\hline 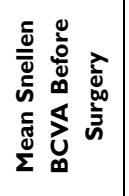 & $\mathbb{Z}$ & $\stackrel{\circ}{\circ}$ & 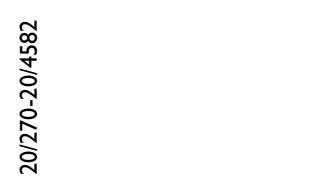 & $\stackrel{\circ}{\circ}$ & 茅 \\
\hline 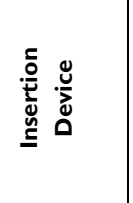 & 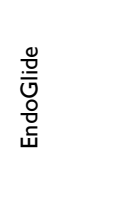 & 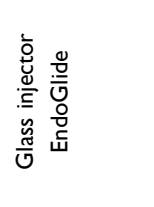 & 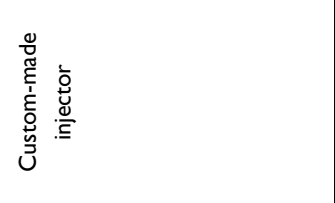 & 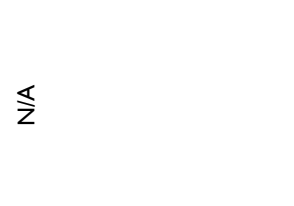 & 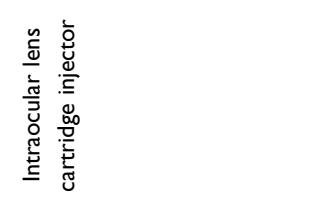 \\
\hline 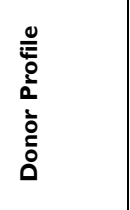 & 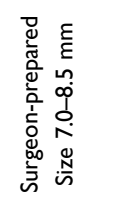 & 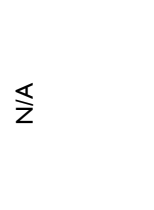 & 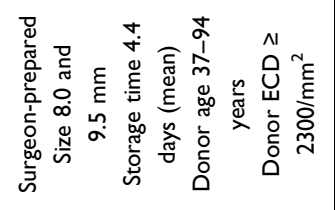 & 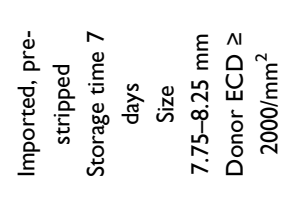 & 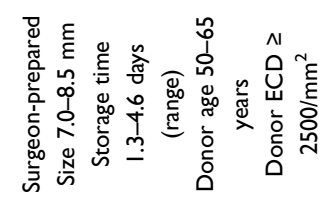 \\
\hline$\frac{2}{\frac{\sigma}{2}}$ & 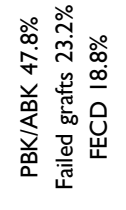 & 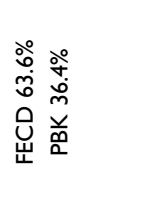 & 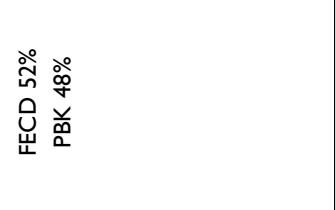 & 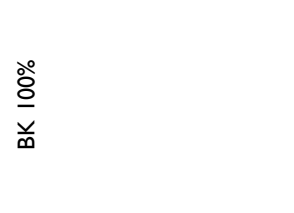 & 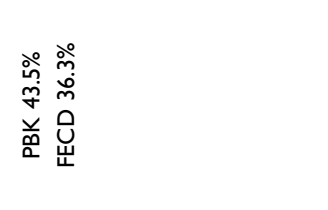 \\
\hline 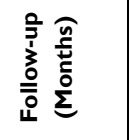 & 0 & $\sigma$ & $\circ$ & $\frac{\pi}{\Lambda}$ & 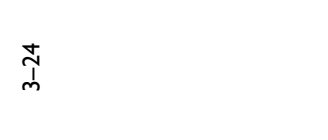 \\
\hline$\dot{z}$ z & వి & $\overline{\underline{I}}$ & $\underline{8}$ & $N$ & \& \\
\hline 密 & 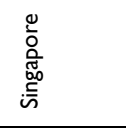 & 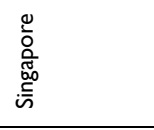 & 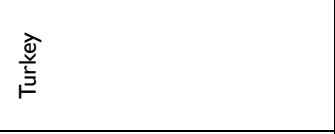 & 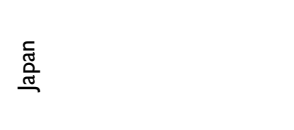 & $\stackrel{\underline{\underline{m}}}{\underline{\underline{\underline{z}}}}$ \\
\hline 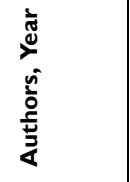 & 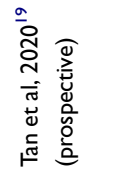 & 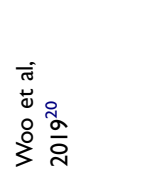 & 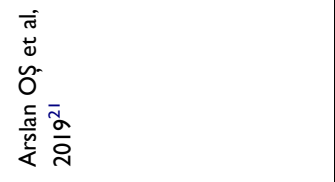 & 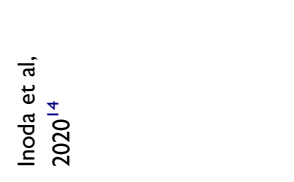 & 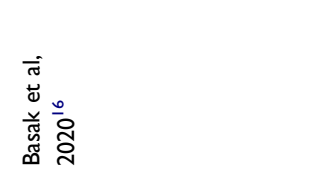 \\
\hline
\end{tabular}




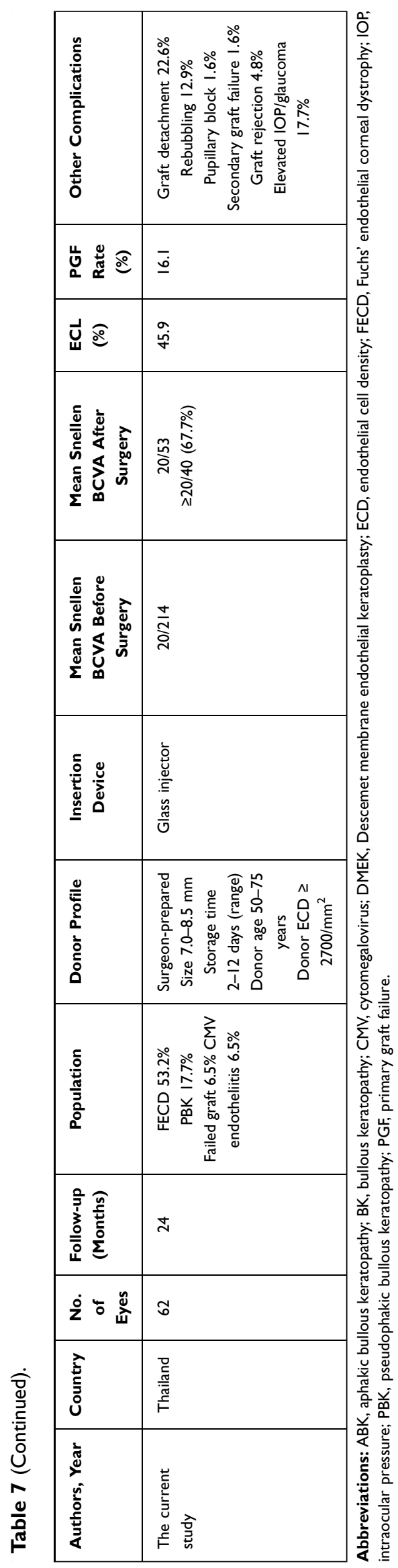

DMEK. The rate of ECL then reduced after 3 months. Although the pattern and degree of ECL in this study corresponded to what were observed in prior non-Asian and Asian studies, ${ }^{1,14,16,34,35}$ there was a small spike in the percentage of ECL between the 6-month and 12-month follow-up in this study. The rise in the rate of ECL might be attributed to postoperative complications such as endothelial rejection, recurrent CMV endotheliitis, and IOP elevation. In addition, graft diameter was the only factor that correlated with postoperative ECD. This finding was also observed in Japanese eyes with bullous keratopathy. ${ }^{14}$ Conversely, the study from Europe reported that postoperative ECD was not significantly associated with DMEK graft sizes in the range of $8-10 \mathrm{~mm}$ in FECD cases with healthy peripheral host endothelium. ${ }^{36}$ This is likely due to smaller eyeballs with shallower anterior chambers in Asians, causing smaller graft sizes used in our study $(7.0-8.5 \mathrm{~mm})$ and Japanese study $(5.0-8.5 \mathrm{~mm}) .{ }^{14}$ More importantly, advanced FECD and bullous keratopathy account for higher proportion of EK cases in Asia. ${ }^{3,20}$ Therefore, using a sufficiently large graft diameter, theoretically yielding a higher endothelial cell count, but not too large when performing DMEK in Asian eyes may be advisable to balance the benefit of having better endothelial survival and the risk of encountering intraoperative difficulties in unfolding and positioning graft as well as postoperative re-bubbling.

Donor ECD, donor storage time, preoperative diagnosis, axial length, surgical technique, postoperative graft detachment, and re-bubbling have been identified as certain factors associated with postoperative ECL. ${ }^{2,13,14,18,35,37-40}$ Nevertheless, there were no signification relationships between these factors and ECL in the current study. This was possibly because some of these factors have been controlled to be least variable including donor ECD and surgical technique. Also, although FECD was the most frequent indication for DMEK, it represented only half of our patients and most cases presented with advanced disease. Thus, 24-month postoperative ECD for FECD eyes resembled those for non-FECD eyes in this study. Additionally, the average axial length in our study was $23.3 \mathrm{~mm}$, with only 4 eyes having the axial length of $\geq 24.5 \mathrm{~mm}$. Hence, the impact of axial length on ECL might not be obvious, similarly to the results from Japanese patients. ${ }^{14}$ As for postoperative graft detachment and re-bubbling, postoperative ECD could not be evaluated in most of our patients with graft detachment needing 
re-bubbling, because they ended up with primary graft failure.

Regarding the concentration and exposure time of trypan blue and ECL, there have been a few in vitro studies attempting to determine the proper concentration and the duration of application of trypan blue dye to DMEK donor corneal tissue to enhance visibility while minimizing endothelial toxicity. ${ }^{29,30}$ Staining DM grafts with $0.06 \%$ trypan blue (VisionBlue ${ }^{\circledR}$, DORC International, Zuidland, The Netherlands), which is often used in DMEK, up to 5 minutes was found to be effective and safe. ${ }^{29}$ At the same time, treating of DM tissue with the higher concentration of $0.15 \%$ trypan blue (MembraneBlue ${ }^{\circledR}$, DORC International, Zuidland, The Netherlands), typically used for vitreoretinal surgery, up to 3-4 minutes led to a deeper blue stain for a prolonged period without detrimental effects on endothelial cell viability. ${ }^{29,30}$ However, a longer staining time of 5 minutes with $0.15 \%$ concentration resulted in a decrease in ECD, approaching statistical significance. $^{29}$ In general, a DMEK graft is clinically stained with $0.06 \%$ trypan blue for about 30 seconds to 5 minutes before transplantation. ${ }^{23,31,41}$ This usually allows the surgeon to clearly visualize the donor tissue in the recipient anterior chamber and exhibits no endothelial toxicity. Nevertheless, in this study, a 3-minute stain with $0.1 \%$ trypan blue was chosen because our patients had a dark iris and most of them had long-standing corneal edema, further compromising intraoperative graft visualization. Moreover, given that each surgeon performed fewer than 50 DMEK surgeries at the time of data collection, the process of graft orientation, unscrolling, and positioning might not be as fast as those conducted by highly experienced surgeons. Interestingly, the rates of postoperative ECL in this study were not different from those reported in the series using $0.06 \%$ concentration. $^{1,14,16,34,35}$ This clinical observation, supported by the previous laboratory findings, suggests that the clinical application of higher concentration of $0.1 \%$ trypan blue for up to 3 minutes ensures graft visibility for a sufficient time with no adverse effect on medium-term ECL.

Postoperative complications and their incidence mostly found in this study were not different from those reported in previous studies. ${ }^{1,42}$ Graft detachment was the major postoperative complication (22.6\%) and $12.9 \%$ underwent re-bubbling procedures. However, our study showed an unusually high rate of primary graft failure, detected in $16.1 \%$ of eyes. Generally, as a result of standardizing surgical techniques, primary graft failure rates reduced from as high as $20 \%$ in initial series to as low as $0 \%$ in recent series. ${ }^{42-44}$ The average rate was $1.9 \%$ (range $0-12.5 \%$ ). ${ }^{1}$ Probably due to its low incidence, there has been no study on risk factors for primary graft failure after DMEK. In this study, we excluded the first 10 cases and used the same standardized "no-touch" technique to minimize the initial learning-curve effects and the impact of different surgical techniques. Nonetheless, 3 cases had intraoperative complications and all of them developed primary graft failure. Due to the small number, our univariate analysis revealed no statistically significant correlation between primary graft failure and presence of intraoperative complications. However, DMEK procedure is relatively new, and we have less experience with it than with PK and DSAEK. Therefore, primary graft failure might still be linked to the surgical learning curve. Meanwhile, in cases with uneventful surgery, other factors must play a role. Deathto-operation time and the diagnosis of PBK were identified as significant predisposing factors for primary graft failure. A plausible explanation is that the Optisol-GS storage time in this study was extremely long (mean, 7.6 days; range, 2-12 day), as 70\% of donor corneas were imported tissues with a mean storage time of 9.0 days (range, 7-12 days). Normally, the death-to-surgery time of DMEK donor tissues ranged from 1.2 to 10 days, with an average of no longer than 7 days. ${ }^{45,46}$ Previous studies demonstrated that ECD of corneas stored in Optisol-GS decreased steadily and probably dropped below the critical level of 2200 cells $/ \mathrm{mm}^{2}$ after 9 days of storage; ${ }^{47}$ and the postoperative endothelial cell loss largely occurs in the early period owing to iatrogenic trauma from graft manipulation. ${ }^{1}$ Thus, using long stored donor tissues with borderline ECD may render endothelial cells more susceptible to surgical trauma, potentially posing a risk for primary graft failure to patients. Re-evaluating tissues stored in Optisol-GS for greater than 7 days by repeat specular microscopy prior to surgery is recommended if the equipment available. As for the original diagnosis, whereas FECD eyes perform better in terms of endothelial cell rehabilitation, which could be attributed to a conserved integrity or regenerative capacity of peripheral endothelial cells in the recipient cornea, PBK eyes may respond less favorable because of lacking host endothelial cells and pathologic changes at the level of the corneal endothelium and/or the stroma. ${ }^{37}$ Hence, considering using DMEK grafts of premium quality such as 
grafts with a higher preoperative ECD or short preservation time may enhance endothelial cell rehabilitation and reduce the likelihood of primary graft failure in PBK cases. Nevertheless, it should be emphasized that neither the independent associations nor causal relationships between death-to-operation time or the diagnosis of PBK and primary graft failure could be claimed because adjusting for multiple other potential predicting factors was not able to be carried out.

The limitations of our study included the small sample size, heterogeneous patient populations, multiple surgeons, short follow-up time, and inherent disadvantages of retrospective study.

In conclusion, our study suggests that despite specific technical challenges, DMEK is a safe, effective, and feasible treatment for endothelial failure in Asian eyes. A larger graft size is crucial in maintaining postoperative endothelial cell survival. Careful case selection, use of relatively fresh donor tissues, and appropriate surgical techniques can prevent primary graft failure and facilitate optimal outcomes following surgery.

\section{Statement of Ethics}

Written informed consent was obtained from all patients.

\section{Funding}

There is no funding to report.

\section{Disclosure}

The authors declare that they have no conflicts of interest in this work.

\section{References}

1. Deng SX, Lee WB, Hammersmith KM, et al. Descemet membrane endothelial keratoplasty: safety and outcomes: a report by the American Academy of Ophthalmology. Ophthalmology. 2018;125:295-310. doi:10.1016/j.ophtha.2017.08.015.

2. Schaub F, Collmer M, Schrittenlocher S, Bachmann BO, Cursiefen C, Hos D. Outcome of Descemet membrane endothelial keratoplasty using corneas from donors $\geq 80$ years of age. Am J Ophthalmol. 2020;211:200-206. doi:10.1016/j.ajo.2019.12.001.

3. Mohamed A, Chaurasia S, Murthy SI, et al. Endothelial keratoplasty: a review of indications at a tertiary eye care centre in South India. Asia Pac J Ophthalmol (Phila). 2014;3:207-210. doi:10.1097/ APO.0b013e3182a75304.

4. Young AL, Kam KW, Jhanji V, Cheng LL, Rao SK. A new era in corneal transplantation: paradigm shift and evolution of techniques. Hong Kong Med J. 2012;18:509-516.

5. Tan DT, Anshu A, Mehta JS. Paradigm shifts in corneal transplantation. Ann Acad Med Singapore. 2009;38:332-338.

6. Ali Javadi M, Kanavi MR, Safi S. A 27-year report from the Central Eye Bank of Iran. J Ophthalmic Vis Res. 2020;15:149-159. doi:10.18502/jovr.v15i2.6731.
7. Bozkurt TK, Acar B, Kilavuzoglu AE, et al. An 11-year review of keratoplasty in a tertiary referral center in Turkey: changing surgical techniques for similar indications. Eye Contact Lens. 2017;43:364-370. doi:10.1097/ICL.0000000000000274.

8. Zare M, Javadi MA, Einollahi B, et al. Changing indications and surgical techniques for corneal transplantation between 2004 and 2009 at a tertiary referral center. Middle East Afr J Ophthalmol. 2012;19:323-329. doi:10.4103/0974-9233.97941.

9. Al-Arfai KM, Yassin SA, Al-Beshri AS, Al-Jindan MY, Al-Tamimi ER. Indications and techniques employed for keratoplasty in the Eastern province of Saudi Arabia: 6 years of experience. Ann Saudi Med. 2015;35:387-393. doi:10.5144/0256-4947.2015.387.

10. Hayashi T, Oyakawa I, Kato N. Techniques for learning Descemet membrane endothelial keratoplasty for eyes of Asian patients with shallow anterior chamber. Cornea. 2017;36:390-393. doi:10.1097/ ICO.0000000000001093.

11. Shimizu T, Hayashi T, Yuda K, et al. Short axial length and iris damage are associated with iris posterior synechiae after Descemet membrane endothelial keratoplasty in Asian eyes. Cornea. 2018;37:1355-1359. doi:10.1097/ICO.0000000000001698.

12. Inoda S, Hayashi T, Takahashi H, et al. Risk factors for cystoid macular edema after Descemet membrane endothelial keratoplasty. Cornea. 2019;38:820-824. doi:10.1097/ICO.0000000000001950.

13. Borroni D, Ferronato M, Krumina Z, Parekh M. Importance of axial length and functional corneal endothelial cells in Descemet membrane endothelial keratoplasty. Cornea. 2017;36:e35-e36. doi:10.1097/ICO.0000000000001385.

14. Inoda S, Hayashi T, Takahashi $\mathrm{H}$, et al. Factors associated with endothelial cell density loss post Descemet membrane endothelial keratoplasty for bullous keratopathy in Asia. PLoS One. 2020;15: e0234202. doi:10.1371/journal.pone.0234202.

15. Kobashigawa Y, Shimizu T, Hayashi T, et al. Iris posterior synechiae after Descemet membrane endothelial keratoplasty in Asian eyes: prevention and management of posterior synechiae. Eye Contact Lens. 2020;46:116-120. doi:10.1097/ICL.0000000000000 616.

16. Basak SK, Basak S, Gajendragadkar N, Ghatak M. Overall clinical outcomes of Descemet membrane endothelial keratoplasty in 600 consecutive eyes: a large retrospective case series. Indian $J$ Ophthalmol. 2020;68:1044-1053. doi:10.4103/ijo.IJO_1563 19.

17. Bhandari V, Reddy JK, Relekar K, Prabhu V. Descemet's stripping automated endothelial keratoplasty versus Descemet's membrane endothelial keratoplasty in the fellow eye for Fuchs endothelial dystrophy: a retrospective study. Biomed Res Int. 2015;2015:750567. doi:10.1155/2015/750567.

18. Ang M, Ting DSJ, Kumar A, May KO, Htoon HM, Mehta JS. Descemet membrane endothelial keratoplasty in Asian eyes: intraoperative and postoperative complications. Cornea. 2020;39:940-945. doi:10.1097/ICO.0000000000002302.

19. Tan TE, Devarajan K, Seah XY, et al. Descemet membrane endothelial keratoplasty with a pull-through insertion device: surgical technique, endothelial cell loss, and early clinical results. Cornea. 2020;39:558-565. doi:10.1097/ICO.0000000000002268.

20. Woo JH, Ang M, Htoon HM, Tan D. Descemet membrane endothelial keratoplasty versus Descemet stripping automated endothelial keratoplasty and penetrating keratoplasty. Am J Ophthalmol. 2019;207:288-303. doi:10.1016/j.ajo.2019.06.012.

21. Arslan OŞ, Doğan C, Mergen B. Six-month results of Descemet membrane endothelial keratoplasty in 100 eyes: first clinical results from Turkey. Turk J Ophthalmol. 2019;49:235-242. doi:10.4274/tjo. galenos.2019.27813.

22. Koçluk Y, Kasım B, Sukgen EA, Burcu A. Descemet membrane endothelial keratoplasty (DMEK): intraoperative and postoperative complications and clinical results. Arq Bras Oftalmol. 2018;81:212-218. doi:10.5935/0004-2749.20180043. 
23. Singh SK, Sitaula S. Visual outcome of Descemet membrane endothelial keratoplasty during the learning curve in initial fifty cases. J Ophthalmol. 2019;2019:5921846. doi:10.1155/2019/5921846.

24. Lekhanont K, Vanikieti K, Nimvorapun N, Chuckpaiwong V. Outcomes of Descemet stripping automated endothelial keratoplasty using imported donor corneas. BMC Ophthalmol. 2017;17:41. doi:10.1186/s12886-017-0436-0.

25. Price MO, Giebel AW, Fairchild KM, Price FW Jr. Descemet's membrane endothelial keratoplasty: prospective multicenter study of visual and refractive outcomes and endothelial survival. Ophthalmology. 2009;116:2361-2368. doi:10.1016/j. ophtha.2009.07.010.

26. Guerra FP, Anshu A, Price MO, Giebel AW, Price FW. Descemet's membrane endothelial keratoplasty: prospective study of 1-year visual outcomes, graft survival, and endothelial cell loss. Ophthalmology. 2011;118:2368-2373. doi:10.1016/j. ophtha.2011.06.002.

27. Ham L, Dapena I, Moutsouris K, et al. Refractive change and stability after Descemet membrane endothelial keratoplasty. Effect of corneal dehydration-induced hyperopic shift on intraocular lens power calculation. J Cataract Refract Surg. 2011;37:1455-1464. doi:10.1016/j.jcrs.2011.02.033.

28. Schoenberg ED, Price FW Jr, Miller J, McKee Y, Price MO. Refractive outcomes of Descemet membrane endothelial keratoplasty triple procedures (combined with cataract surgery). J Cataract Refract Surg. 2015;41:1182-1189. doi:10.1016/j.jcrs.2014.09.042.

29. Majmudar PA, Johnson L. Enhancing DMEK success by identifying optimal levels of trypan blue dye application to donor corneal tissue. Cornea. 2017;36:217-221. doi:10.1097/ICO.0000000000001074

30. Weber IP, Rana M, Thomas PBM, Dimov IB, Franze K, Rajan MS. Effect of vital dyes on human corneal endothelium and elasticity of Descemet's membrane. PLoS One. 2017;12:e0184375. doi:10.1371/ journal.pone.0184375.

31. Dapena I, Moutsouris K, Droutsas K, Ham L, van Dijk K, Melles GR. Standardized "no-touch" technique for descemet membrane endothelial keratoplasty. Arch Ophthalmol. 2011;129:88-94. doi:10.1001/archophthalmol.2010.334.

32. Chen SY, Terry MA. Step-by-step Descemet's membrane endothelial keratoplasty surgery. Taiwan J Ophthalmol. 2019;9:18-26. doi:10.4103/tjo.tjo_108_18.

33. Jacob S, Agarwal A, Kumar DA. Endoilluminator-assisted Descemet membrane endothelial keratoplasty and endoilluminator-assisted pre-Descemet endothelial keratoplasty. Clin Ophthalmol. 2015;9:2123-2125. doi:10.2147/OPTH.S95282.

34. Vasiliauskaitė I, Oellerich S, Ham L, et al. Descemet membrane endothelial keratoplasty: ten-year graft survival and clinical outcomes. Am J Ophthalmol. 2020;217:114-120. doi:10.1016/j. ajo.2020.04.005.

35. Peraza-Nieves J, Baydoun L, Dapena I, et al. Two-year clinical outcome of 500 consecutive cases undergoing Descemet membrane endothelial keratoplasty. Cornea. 2017;36:655-660. doi:10.1097/ ICO.0000000000001176.
36. Schrittenlocher S, Bachmann B, Cursiefen C. Impact of donor tissue diameter on postoperative central endothelial cell density in Descemet membrane endothelial keratoplasty. Acta Ophthalmol. 2019;97:e618-e622. doi:10.1111/aos.13943.

37. Birbal RS, Baydoun L, Ham L, et al. Effect of surgical indication and preoperative lens status on Descemet membrane endothelial keratoplasty outcomes. Am J Ophthalmol. 2020;212:79-87. doi:10.1016/j. ajo.2019.12.011.

38. Baydoun L, Ham L, Borderie V, et al. Endothelial survival after Descemet membrane endothelial keratoplasty: effect of surgical indication and graft adherence status. JAMA Ophthalmol. 2015;133:1277-1285. doi:10.1001/jamaophthalmol.2015.3064.

39. Rodríguez-calvo de Mora M, Groeneveld-van Beek EA, Frank LE, et al. Association between graft storage time and donor age with endothelial cell density and graft adherence after Descemet membrane endothelial keratoplasty. JAMA Ophthalmol. 2016;134:91-94. doi:10.1001/jamaophthalmol.2015.4499.

40. Hayashi T, Schrittenlocher S, Siebelmann S, et al. Risk factors for endothelial cell loss after Descemet membrane endothelial keratoplasty (DMEK). Sci Rep. 2020;10:11086. doi:10.1038/s41598-02068023-0

41. Mori N, Yokogawa H, Kobayashi A, Nishino T, Sugiyama K. Surgery-induced iris abnormalities after Descemet membrane endothelial keratoplasty and their impact on postoperative clinical outcomes. Clin Ophthalmol. 2019;13:805-809. doi:10.2147/OPTH. S196906.

42. Ang M, Wilkins MR, Mehta JS, Tan D. Descemet membrane endothelial keratoplasty. $\mathrm{Br} J$ Ophthalmol. 2016;100:15-21. doi:10.1136/bjophthalmol-2015-306837.

43. Dapena I, Ham L, Droutsas K, van Dijk K, Moutsouris K, Melles GR. Learning curve in Descemet's membrane endothelial keratoplasty: first series of 135 consecutive cases. Ophthalmology. 2011;118:2147-2154. doi:10.1016/j.ophtha.2011.03.037.

44. Monnereau C, Quilendrino R, Dapena I, et al. Multicenter study of descemet membrane endothelial keratoplasty: first case series of 18 surgeons. JAMA Ophthalmol. 2014;132:1192-1198. doi:10.1001/ jamaophthalmol.2014.1710.

45. Straiko MD, Bauer AJ, Straiko MMW, et al. Donor DMEK tissue characteristics: association with rebubble rate and 6-month endothelial cell loss. Cornea. 2020;39:1267-1273. doi:10.1097/ ICO.0000000000002398.

46. Feng MT, Burkhart ZN, Price FW Jr, Price MO. Effect of donor preparation-to-use times on Descemet membrane endothelial keratoplasty outcomes. Cornea. 2013;32:1080-1082. doi:10.1097/ ICO.0b013e318292a7e5.

47. Sibayan SA, Garcia-Arenal MC, Corpus KD, et al. Serial endothelial cell count of donor corneal buttons in Optisol-GS. Procedia Chem. 2015;14:394-397. doi:10.1016/j.proche.2015.03.053.
Clinical Ophthalmology

\section{Publish your work in this journal}

Clinical Ophthalmology is an international, peer-reviewed journal covering all subspecialties within ophthalmology. Key topics include: Optometry; Visual science; Pharmacology and drug therapy in eye diseases; Basic Sciences; Primary and Secondary eye care; Patient Safety and Quality of Care Improvements. This journal is indexed on PubMed
Central and CAS, and is the official journal of The Society of Clinical Ophthalmology (SCO). The manuscript management system is completely online and includes a very quick and fair peer-review system, which is all easy to use. Visit http://www.dovepress.com/ testimonials.php to read real quotes from published authors. 